\title{
(6) OPEN ACCESS \\ An update on the use and investigation of probiotics in health and disease
}

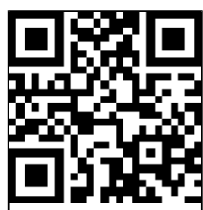

Editor's choice can to access mo free content

${ }^{1}$ Dairy \& Food Culture Technologies, Centennial, Colorado, USA

${ }^{2}$ Digestive System Research Unit, University Hospital Vall d'Hebron, CIBEREHD, Barcelona, Spain

${ }^{3}$ Center for Global Health, University of Virginia School of Medicine, Charlottesville, Virginia, USA

${ }^{4}$ Laboratory of Biochemical Genetics \& Metabolism, The Rockefeller University,

New York City, New York, USA ${ }^{5}$ Department of Medicine,

Alimentary Pharmabiotic Centre, University College Cork, Ireland

${ }^{6}$ Division of Gastroenterology and Hepatology, The Methodist Hospital and Weill Cornell School of Medicine, Houston, Texas, USA

${ }^{7}$ Departments of Medicine Microbiology and Immunology, University of North Carolina at Chapel Hill School of Medicine, Chapel Hill, North Carolina, USA

${ }^{8}$ The Hospital for Sick Children, University of Toronto, Toronto, Ontario, Canada

${ }^{9}$ Division of Digestive Diseases, University of California, Los Angeles, California, USA

\section{Correspondence to}

Emeran A Mayer, Oppenheimer Center for Neurobiology of

Stress, Division of Digestive Diseases, UCLA CHS 47-122, 10833 Le Conte Ave, Los Angeles, CA 90095-7378, USA;emayer@ucla.edu

Received 16 May 2012 Revised 5 February 2013 Accepted 7 February 2013

Published Online First

8 March 2013

\author{
Mary Ellen Sanders, ${ }^{1}$ Francisco Guarner, ${ }^{2}$ Richard Guerrant, ${ }^{3}$ Peter R Holt, \\ Eamonn MM Quigley, ${ }^{5,6}$ R Balfour Sartor, ${ }^{7}$ Philip M Sherman, ${ }^{8}$ \\ Emeran A Mayer ${ }^{9}$
}

\begin{abstract}
Probiotics are derived from traditional fermented foods, from beneficial commensals or from the environment. They act through diverse mechanisms affecting the composition or function of the commensal microbiota and by altering host epithelial and immunological responses. Certain probiotic interventions have shown promise in selected clinical conditions where aberrant microbiota have been reported, such as atopic dermatitis, necrotising enterocolitis, pouchitis and possibly irritable bowel syndrome. However, no studies have been conducted that can causally link clinical improvements to probiotic-induced microbiota changes. Whether a disease-prone microbiota pattern can be remodelled to a more robust, resilient and disease-free state by probiotic administration remains a key unanswered question. Progress in this area will be facilitated by: optimising strain, dose and product formulations, including protective commensal species; matching these formulations with selectively responsive subpopulations; and identifying ways to manipulate diet to modify bacterial profiles and metabolism.
\end{abstract}

\section{INTRODUCTION}

'If gut bacteria are making you ill, can swapping them make you healthy?' asks an article from The Economist (18 August 2012, 'Me, myself, us', p69). This is where the concept of probiotics enters the discussion about microbiota gone awry. Probiotics are live micro-organisms, which, when administered in adequate amounts, confer a health benefit to the host. Probiotics act through diverse mechanisms that affect the microbiota. ${ }^{1} 2$ This effect may be revealed through changes in either the populations of bacteria or bacterial metabolic activity. A recent study demonstrated that a probiotic yogurt changed urinary bacterial metabolites, but not faecal bacterial community populations. ${ }^{3}$ Such results suggest that probiotics may have the potential to affect the function more than the structure of the microbiome. Probiotics are the subject of increasing basic and clinical research, while also being incorporated into an expanding array of foods, nutritional supplements and pharmaceutical products globally (figure 1).

The literature on the health benefits of probiotics has often focused on disease states using either animal models of such diseases or studies in human populations. ${ }^{4}$ More recently, investigators have been asking how to measure the impact of probiotics on healthy individuals, such as reducing the risk of developing disease or optimising physiological function within normal ranges. The distinction between research aimed at maintaining health and that aimed at treating a disease has important regulatory implications; the former can be applied to foods and supplements, whereas the latter is confined to drugs.

This review provides an update on probiotic effects on treatment or prevention of important gastroenterological conditions: irritable bowel syndrome (IBS), infectious diarrhoea including nosocomial infections, inflammatory bowel disease (IBD), necrotising enterocolitis (NEC), as well as cancer and cancer therapy. We also address the impact of probiotics on indicators of health, as measured through reduction in the incidence of common infectious diseases and risk of allergy, improvement in bowel function, and modulation of immune function. When available, conclusions arising from meta-analyses or systematic reviews on probiotic effects are provided. Lastly, we highlight challenges and opportunities in conducting human research in this field.

\section{PROBIOTICS IN HEALTH AND DISEASE Irritable bowel syndrome}

IBS is one of the most common intestinal disorders in industrialised (affecting 10-15\% of the population) and developing countries and incurs significant healthcare costs. ${ }^{5} 6$ In the absence of generally agreed upon biomarkers, IBS is currently defined by symptom criteria, which include chronic recurring episodes of abdominal pain or discomfort associated with altered bowel habits in the absence of organic disease. ${ }^{7}$ In addition, sensations of bloating with and without visible abdominal distension, increased trait anxiety and several extraintestinal symptoms commonly occur. ${ }^{5} 78$ Since IBS-like symptoms can also occur in a milder form in healthy individuals, studies on subjects with IBS are relevant to the general, undiagnosed population, as reflected in the European regulatory framework. ${ }^{9}$ Although preliminary evidence suggests alterations in gut microbiota in patients with 


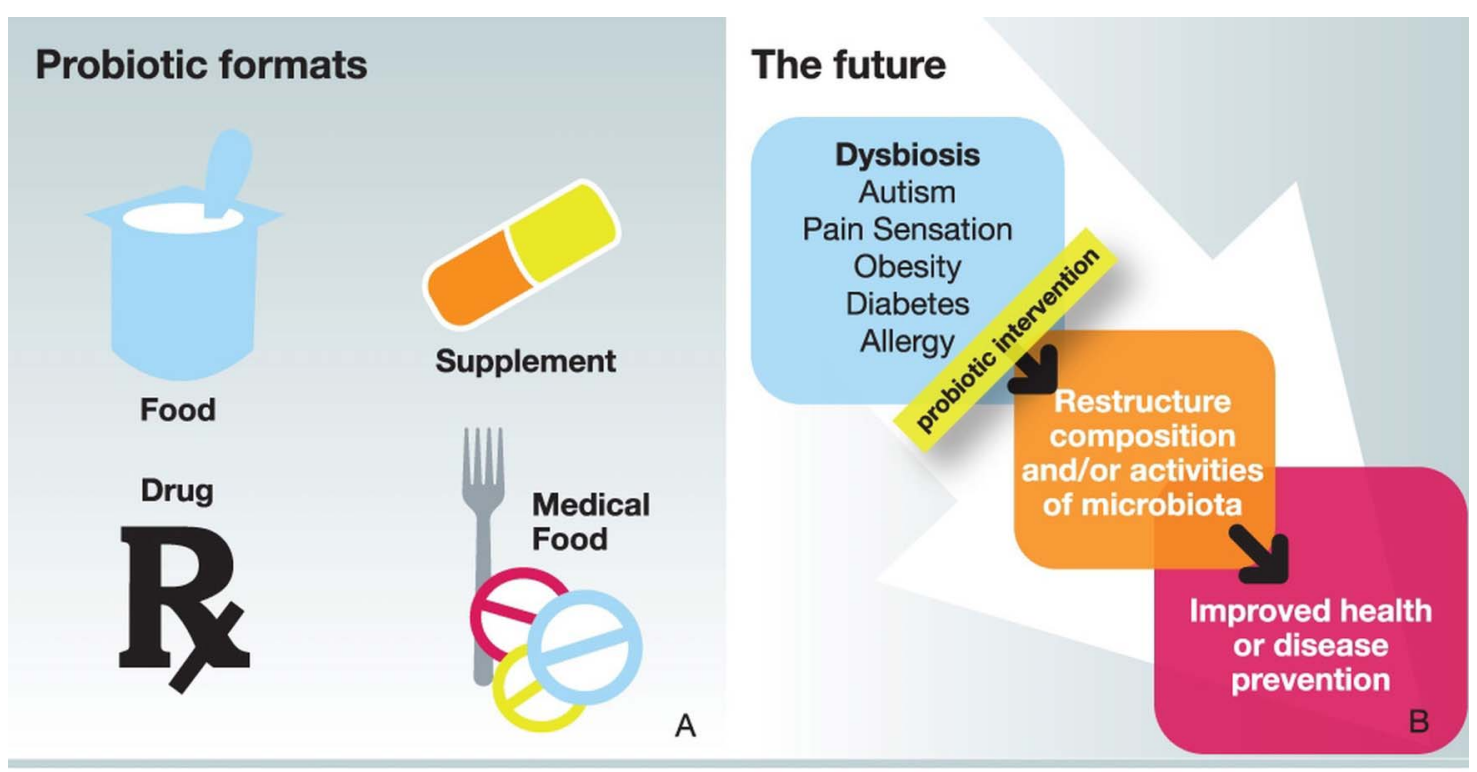

\section{Health and clinical targets addressed by studies on orally administered probiotics conducted in human subjects of different ages}
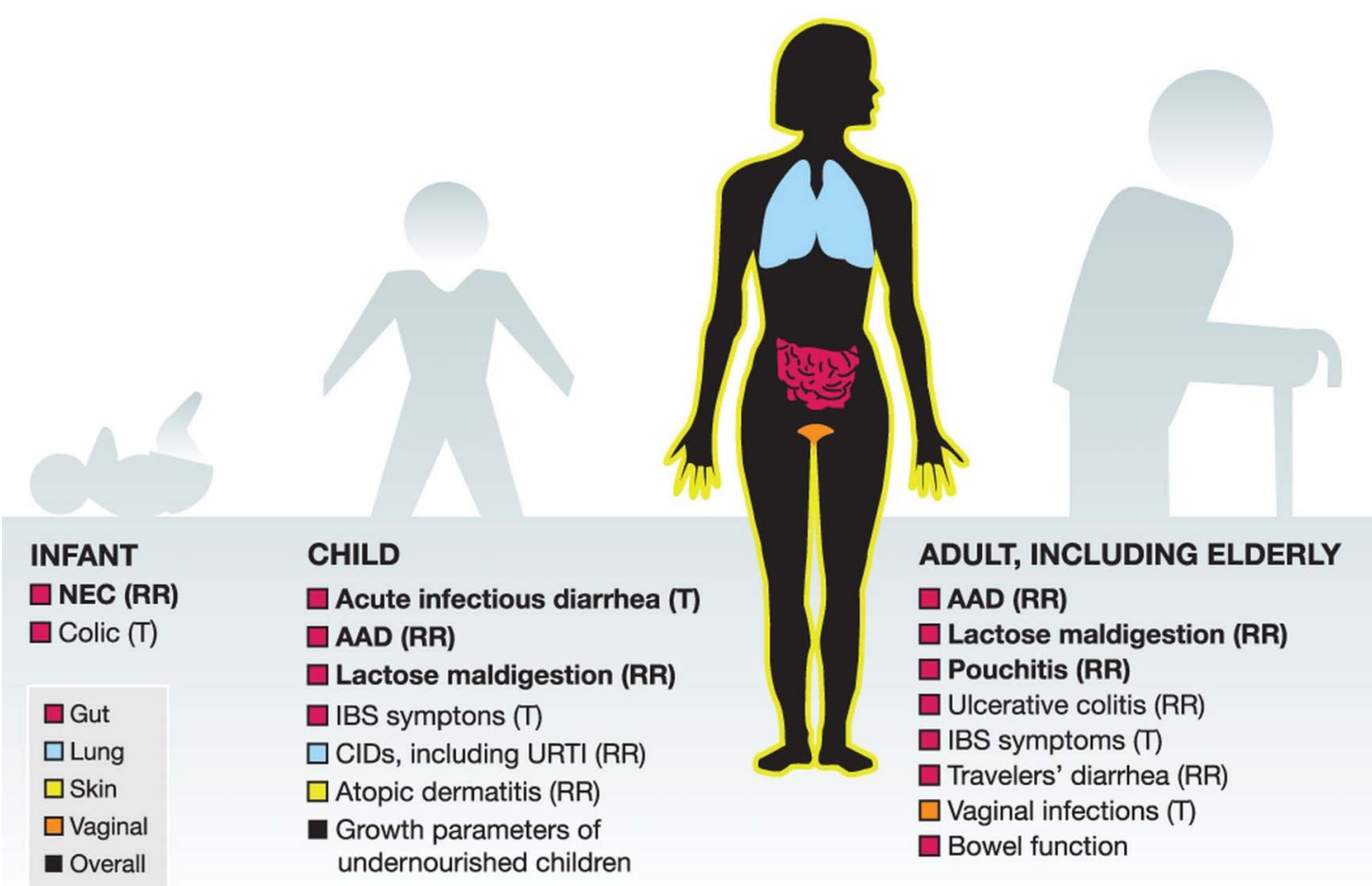

C

Figure 1 Scope of probiotic products and uses. (A) Probiotics can be found in food, dietary/nutritional supplements, drugs and medical foods. Each product has country-specific legal requirements for allowed claims of efficacy, target populations, safety and risk/benefit assessment. (B) Hypothesised future uses for probiotics in modifying the composition or activities of the microbiota for improved health. (C) A range of health and clinical targets for different probiotics have been studied, encompassing intestinal and extraintestinal sites, and over a range of life stages. The evidence is strongest in the conditions shown in bold. Mechanisms for observed health effects may not be known, but probably include direct or indirect action on the activities and/or populations of gut microbiota and on the intestinal immune system. AAD, antibiotic-associated diarrhoea; CID, common infectious disease; IBS, irritable bowel syndrome; NEC, necrotising enterocolitis; RR, reduced risk; T, treatment; URTI, upper respiratory tract infections.

IBS, ${ }^{10-15}$ it remains to be determined if these alterations are a cause or a consequence of altered gut motility and secretion. ${ }^{16}$ Recent preclinical data support the concept that gut microbiota and probiotics affect enteric nervous system and brain signalling; beneficial effects of probiotics on visceral nociceptive reflexes in rodents have also been described. ${ }^{17}$ However, only preliminary data suggest that such mechanisms may also play a role in healthy subjects or those with IBS. ${ }^{18}$

A growing number of meta-analyses vary in their conclusions on the effectiveness of probiotics against IBS, in part because 
of inadequate sample size, poor study design and use of various probiotic strains in the reviewed studies. ${ }^{19-21}$ Moayyedi et al ${ }^{19}$ reviewed 19 randomised, controlled trials (RCTs) performed in 1650 patients with IBS and concluded that probiotics were better than placebo (relative risk of IBS not improving 0.71 (95\% CI 0.57 to 0.88 ) with a number needed to treat of $4(95 \%$ CI 3.0 to 12.5)). Clarke et al ${ }^{20}$ reviewed 42 RCTs of the effect of lactic acid bacteria probiotics on IBS symptoms. Thirty-four of these trials reported benefit in at least one of the end points studied. Brenner et al ${ }^{21}$ evaluated 16 strictly selected RCTs and found 11 that were inadequately blinded, of too short duration, of too small sample size, and/or lacked intention to treat analysis. They concluded that only two of the studies-those using Bifidobacterium infantis $35624^{22} 23$-showed significant improvements in abdominal pain/discomfort, bloating/distension and/or bowel movements compared with placebo. Given the controversies in IBS pathophysiology, patient heterogeneity, or lack of clear, reproducible evidence for gut microbiota abnormalities in patients with IBS, additional RCTs with appropriate end points and design are needed to determine the extent to which (and in which IBS subpopulations) certain probiotics are useful therapeutic strategies in the management of IBS symptoms.

\section{Infectious diarrhoea}

Enteric and diarrhoeal diseases are leading causes of morbidity and mortality among children under the age of 5 worldwide, with low- and middle-income countries bearing the brunt of this burden. ${ }^{24}$ Repeated infections lead to acute and chronic undernutrition, resulting in more frequent and more severe infections; eventually this leads to developmental deficits in growth, fitness and cognition, which persist into adulthood with devastating human and economic consequences globally. ${ }^{25-27} \mathrm{~A}$ better understanding of the intestinal microbiota and of potential action mechanisms of probiotics has led to studies evaluating their efficacy in acute infectious gastroenteritis $^{28}$ and in the setting of persistent diarrhoea. ${ }^{29}$ Such approaches have the potential to help reduce the global burden of childhood disease. ${ }^{30}$ Treatment of acute diarrhoea with probiotics appears to reduce diarrhoea duration by about 1 day (predominantly in developed areas; 22 studies carried out in developing areas were more variable). ${ }^{25} 28{ }^{31}$ In persistent diarrhoea in developing areas, an approximate 4-day reduction in the duration of persistent diarrhoea, coupled with improved growth parameters, has been noted. ${ }^{25} 2931$

Several studies with probiotics, including Saccharomyces boulardi, Lactobacillus rhamnosus GG and other strains report reductions in nosocomial diarrhoea rates, as well as reductions in antibiotic-associated diarrhoea and recurrences of Clostridium difficile-associated diarrhoea. ${ }^{31}{ }^{32}$ These effects include a $40-60 \%$ reduction in the frequency of antibiotic-associated diarrhoea, but studies documenting a reduction in $C$ difficile-associated diarrhoea are far fewer and remain the subject of controversy. ${ }^{33-36}$ Indeed, Floch et al ${ }^{32}$ considered evidence insufficient for an ' $\mathrm{A}$ ' recommendation for this indication.

Nosocomial infections remain a major healthcare concern, with estimated yearly direct medical costs in the USA of US $\$ 28-45$ billion, ${ }^{37}$ pointing to the need for a preventive approach. However, the results of studies evaluating the preventive effect of probiotics on nosocomial infections have been mixed. Some show benefit, ${ }^{38} 39$ whereas others do not. ${ }^{40-42}$ Three RCTs (including 1043 children) tested L rhamnosus GG supplementation and showed significantly reduced rates of nosocomial rotavirus diarrhoea. ${ }^{39} 42-44$ Supplementing infant formula with $B$ bifidum and Streptococcus thermophilus reduced the frequency of episodes of acute infectious diarrhoea. ${ }^{45} \mathrm{~L}$ rhamnosus GG was effective in reducing nosocomial gastrointestinal and respiratory tract illnesses in over 2000 immunocompetent children $\geq 1$ year of age without underlying illnesses who were suddenly hospitalised for reasons unrelated to gastrointestinal or respiratory tract problems. ${ }^{39}$ Although probiotics show promise in reducing nosocomial infections among some populations, they are not recommended for critically ill hospitalised patients at this time. ${ }^{46} 47$

\section{Inflammatory bowel disease}

Probiotic treatments of IBD have yet to meet the high expectations predicted by mechanistic and animal studies, especially for Crohn's disease. ${ }^{48} 49$ No consistent effects have been noted in treating or preventing relapse of Crohn's disease. For ulcerative colitis, benefits have been described for a combination of Lactobacillus, Bifidobacterium and Streptococcus probiotic species or for Escherichia coli Nissle in inducing and maintaining remission of disease activity in mild to moderately severe ulcerative colitis. ${ }^{50-53}$ Primary prevention of pouchitis and reducing the likelihood of relapse after successful antibiotic treatment has also been successful, receiving an 'A' recommendation by Floch et al. ${ }^{32}$

Possible reasons for the current disparity between therapeutic potential and actual clinical outcomes of probiotic use in IBD are summarised in table 1, which includes proposed strategies to enhance therapeutic outcomes. Although created for IBD, this conceptual framework is relevant to other complex disorders such as IBS, colorectal and gastric cancers, non-alcoholic steatohepatitis and autoimmune diseases. In these diseases, interactions between genetic, microbial and environmental influences lead to heterogeneous phenotypes in patient subsets that are uniquely responsive to specific microbial manipulations. Functions associated with the over 160 genetic polymorphisms associated with IBD can be broadly grouped as defective mucosal barrier function/healing, abnormal immunoregulation and defective microbial recognition/killing Immunosuppression in a patient with defective bacterial killing may be counterproductive. Likewise an individual with

Table 1 Possible explanations and proposed solutions for disappointing therapeutic results of probiotic treatment of IBD and other conditions

\begin{tabular}{|c|c|}
\hline Reason for failure & Proposed solution \\
\hline Wrong targets & $\begin{array}{l}\text { Individualise treatment based on molecular } \\
\text { pattern of dysbiosis }\end{array}$ \\
\hline Wrong probiotic agents & $\begin{array}{l}\text { Use protective commensal enteric } \\
\text { species, which may be more suitable than } \\
\text { probiotics derived from cultured milk or } \\
\text { foods, complex groups of commensal } \\
\text { species or even intact normal bacterial } \\
\text { communities (faecal transplant) }\end{array}$ \\
\hline Targeting incorrect disease mechanisms & $\begin{array}{l}\text { Tailor therapeutic agent to correct } \\
\text { underlying genetic defect/inflammatory } \\
\text { pathway in an individual }\end{array}$ \\
\hline Product not as potent as needed & $\begin{array}{l}\text { Genetically enhance bacterial function } \\
\text { through addition or deletion of bioactive } \\
\text { genes (pharmabiotics) }\end{array}$ \\
\hline $\begin{array}{l}\text { Product not administered at a time in } \\
\text { relation to the disease onset where it }\end{array}$ & $\begin{array}{l}\text { Target therapy to phase of disease } \\
\text { process }\end{array}$ \\
\hline
\end{tabular}
relation to the disease onset where it can be effective

Age of the subject

Tailor therapy to age/developmental stage of individual subject

IBD, inflammatory bowel disease. 
dysbiosis may respond better to selective restoration of protective commensal species, such as Faecalibacterium prausnitzii or Clostridium species, than to exogenous agents such as traditional probiotics. Polymorphisms in IBD-related genes that regulate mucus glycosylation, such as Fut2, which encodes $\alpha 1,2$-fucosyltransferase and is associated with abnormal bacterial profiles, may selectively improve response to alternative bacterial nutritional sources, such as prebiotics. ${ }^{54}$

The extraction or synthesis of molecules derived from probiotic or protective enteric species could also prove useful. For example, immunoactive purified products such as p40 from $L$ rhamnosus GG or polysaccharide A from Bacteroides fragilis with defined biological actions could be synthesised and administered. ${ }^{55} 56$ In addition, bacteria can be engineered to produce interleukin 10, trefoil factors or elafin. ${ }^{57}$ An alternative approach is to identify and develop dietary strategies to selectively enhance the growth and function of endogenous commensals or diminish the activities of detrimental bacteria. For example, prebiotics such as inulin or fructose oligosaccharides increase luminal numbers of Bifidobacterium species and concentrations of protective short-chain fatty acids, which are important metabolic substrates for colonic epithelial cells. In contrast, refined sugars and food additives, such as iron, can increase proliferation of detrimental bacterial species, including $E$ coli, Klebsiella pneumonia and Enterococcus faecalis. ${ }^{58} 59$ These dietary substances could be avoided to provide better maintenance of healthy states.

One potential therapeutic approach to management of IBD might be to induce rapid clinical remission and mucosal healing with corticosteroid and/or biological therapy followed by probiotic and/or prebiotic interventions to sustain remission (table 2). This novel treatment paradigm remains unproven, but is designed to stimulate new directions of clinical and translational research that have the potential to improve therapeutic results while decreasing long-term toxicity and costs.

\section{Necrotising enterocolitis}

Differences in intestinal microbiota of preterm versus term infants may factor into the preterm infants' predisposition to NEC. ${ }^{60}$ The microbiota of infants with NEC differ from that of other low-birth-weight infants, ${ }^{61}$ particularly in decreased Firmicutes and increased gamma proteobacteria. ${ }^{62}$ Deep sequencing studies before the development of the disease suggest that

Table 2 Future microbial and dietary interventions that could have a role in managing IBD and other intestinal inflammatory conditions once clinical remission and mucosal healing is established

\begin{tabular}{ll}
\hline Intervention and rationale & Reference \\
\hline Induce regulatory (protective) immune responses by probiotics, & 56,118 \\
components of commensals such as Clostridium groups IV and XIVA, & \\
bacterial products such as polysaccharide A or dietary retinoic acid. & \\
Regulatory T cells maintain mucosal homoeostasis and can prevent & \\
relapse of inflammation. & 55 \\
Improving mucosal barrier function with probiotics or their products, \\
including p40 from Lactobacillus rhamnosus GG, and short-chain fatty \\
acids produced by Bifidobacterium and Clostridium species, including \\
Faecalibacterium prausnitzii. Short-chain fatty acids such as butyrate \\
that are products of bacterial metabolism of non-absorbed \\
carbohydrates (fibre and prebiotics) are the primary metabolic fuel of \\
colonic epithelial cells. Inflammation develops in the absence of \\
short-chain fatty acids because of epithelial starvation/damage. \\
Decreasing luminal concentrations of antigens and TLR ligands that \\
drive aggressive immune responses. Commensal luminal microbial \\
antigens stimulate the TH1/TH17 responses that mediate chronic \\
inflammation in Crohn's disease.
\end{tabular}

$\mathrm{IBD}$, inflammatory bowel disease. individual operational taxonomic units differ between patients with NEC and controls. ${ }^{63}$ One line of evidence that altered microbiota may predispose infants to the development of NEC is the high prevalence of antibiotic usage in these premature infants.

At the present time, NEC is associated with $30 \%$ mortality, despite extensive medical and surgical efforts, and with severe and costly sequelae if the patient survives. The disease can be difficult to diagnose before intestinal perforation occurs. The immature intestine of preterm infants is especially prone to inflammation and loss of epithelial integrity. ${ }^{64}$ Since probiotics have potential to interfere with this progression, they have been tested clinically for NEC. Indeed, meta-analyses of probiotic studies using strains of Bifidobacterium, Lactobacillus, Saccharomyces and/or $S$ thermophilus to prevent NEC show reduction in the frequency and reduction in overall mortality. ${ }^{65}$ A study from Egypt reported that L rhamnosus GG, or a killed preparation of the same probiotic strain, significantly reduced the incidence of NEC. ${ }^{66}$ Although the American Academy of Pediatrics recognises that there is evidence that probiotics prevent NEC in very-low-birth-weight infants, they call for more studies to clarify the effective dose and strain of probiotic before issuing clinical recommendations. ${ }^{67}$ For example, one systematic review of three RCTs evaluating Bifidobacterium animalis CNCM I-3446 in 293 preterm babies reported only a trend towards prevention of NEC, suggesting that this treatment regimen may not be as effective as others. ${ }^{68}$ Others consider available evidence sufficient to support a change in practice. $^{69}$ This opinion is based on the lack of treatment options for NEC and the strength of evidence that probiotics can prevent severe NEC and all-cause mortality in preterm infants.

\section{Cancer and cancer therapies}

Colorectal cancer (CRC) is one of the most common cancers worldwide. Several molecular and cellular steps in the carcinogenic pathways have been defined, and the body of evidence indicates a prominent causative role for environmental factors, including obesity and diet. Both of these factors are associated with changes in the gut microbiome. It is noteworthy that tumour incidence and mass are greater in conventional than in germ-free rodents. ${ }^{70}$ Taken together, these findings strongly support the concept that the microbiota play some role in CRC, but causality remains to be confirmed. Sears et $a l^{71}$ have presented evidence that enterotoxigenic $B$ fragilis, for example, may trigger $\mathrm{E}$ cadherin catabolism, provoke intestinal inflammation, and thereby increase the risk of colonic cancer. Others have analysed the microbiology in patients with CRC and suggested that the bacterial diversity is less, ${ }^{72}$ altered $^{73} 74$ or accompanied by high levels of Fusobacterium nucleatum sequences. ${ }^{75}$ Studies in rodents have concentrated on probiotic effects on precancerous lesions and tumours. Such studies have yielded consistent, beneficial effects. ${ }^{76} 77$ Several potential mechanisms have been described, including alterations in microbiota species and metabolism, changes in colonic $\mathrm{pH}$, binding or inactivation of carcinogens, enhanced immune responses, reduced colonic inflammation, lowered epithelial proliferation and increased apoptosis. ${ }^{78}$

Biomarker studies in humans show that synbiotics reduced faecal-water-induced genotoxic damage and increased transepithelial resistance. ${ }^{79} 80$ A synbiotic combination of an oligofructose-enriched inulin preparation combined with two probiotics did not affect epithelial cell proliferation ${ }^{81}$ but reduced evidence of faecal-water-induced DNA damage in 
HT29 and colonic epithelial cells. ${ }^{82}$ Rowland and colleagues focused on how administering a prebiotic-probiotic mixture could affect faecal-water genotoxicity in cell culture studies, and demonstrated pronounced within-individual changes in barrier function, immune cell activity, immune cell proliferation and apoptosis. One consistent observation is that a synbiotic preparation appears to be more effective in altering biomarkers of CRC risk than a single probiotic or prebiotic. One human study showed a reduced rate of recurrence of adenoma atypia after 4 years of Lactobacillus casei administration. ${ }^{83}$ Finally, a 12-year follow-up of over 45000 volunteers with a high intake of yogurt in an Italian cohort reported a reduction in CRC, although there was no comparator group in this study. ${ }^{84}$ Although the few human studies conducted on cancer end points in humans are encouraging, the end points are diverse, and findings need to be expanded before clinical recommendations can be considered.

Radiotherapy and chemotherapy, widely used either alone or in combination for the management of intra-abdominal and intrapelvic cancers, kill replicating cells in the rapidly proliferating normal small and large intestine. Probiotics have been evaluated to help manage side effects of these therapies. Germ-free mice and animals in which the microbiota have been modified by antibiotics are more resistant to radiation toxicity, providing a basis for suspecting that interventions targeting the microbiota may be effective. ${ }^{85}{ }^{86}$ L rhamnosus GG and its conditioned medium were found to reduce intestinal damage and apoptosis from radiation in the proximal jejunum of mice in a TLR2-, COX2- and MyD88-dependent fashion. ${ }^{55} 87$ L rhamnosus GG protection appears to be mediated through the unusual mechanism of increased migration of mesenchymal stem cells into the lamina propria. Others also describe the beneficial effects of different probiotics given to patients receiving chemotherapy $^{88} 89$ or radiation. ${ }^{90}$ These and other studies point to the potential beneficial effect of probiotics in the amelioration of radiation and chemotherapy damage to the small bowel and large intestine of patients being treated for cancers. Incapacitating diarrhoea, dehydration and malnutrition are adverse effects of radiotherapy and chemotherapy, which can limit the amounts of therapy that can be administered, thus compromising patient management. ${ }^{91}$ Probiotics that effectively mitigate these side effects of cancer treatment could be important therapeutic agents.

\section{Allergy}

Allergic disorders have been associated with aberrant gut microbiota. ${ }^{92}$ Factors associated with allergy such as birth delivery mode (caesarean section vs vaginal delivery), ${ }^{93}$ antibiotic use in the newborn and infant, and non-breast-milk diets are also associated with shifts in the gut microbiota. In the last several decades, an increasing number of children (approximately 10\% of the general population) develop allergy in a clinical progression of the so-called 'atopic march' (eczema $\rightarrow$ rhinitis $\rightarrow$ asthma). Probiotics have been studied as possible dietary interventions to interrupt this disease progression. A much higher incidence of atopy is described among infants born into a family with an allergic history. For people with such a family history, strategies using probiotics for prevention should begin early, since most studies designed to assess prevention of atopic dermatitis with probiotics have been conducted in the last stages of pregnancy and during lactation. Several studies have shown a persistent and significantly reduced rate of atopic dermatitis for up to 7 years. ${ }^{94} 95$ However, no effect on the expression of asthma later in childhood has been observed. An ideal study would follow allergy-prone compared with non-allergy-prone infants from late pregnancy until late childhood and test for the expression of all forms of allergy, as well as sensitivity, using a standardised protocol. ${ }^{96} 97$ As the situation now stands, evidence of efficacy is not convincing enough to compel paediatric organisations to recommend routine use of probiotics.

\section{Common infectious diseases}

The gut microbiota are being recognised for their role in promoting resistance to non-enteric pathogens, possibly through enhanced barrier function, production of anti-pathogenic substances and improved immune function. ${ }^{98}$ Research reviewed by Borchers et al ${ }^{99}$ suggests that certain probiotics can regulate critical components of the immune system, such as lymphocytes, antibodies and natural killer cells. As an extension of these functions, the potential of probiotics to reduce common infectious diseases has been studied. ${ }^{100}{ }^{101}$ A meta-analysis conducted on the effectiveness of probiotics in preventing acute upper respiratory tract infections (URTIs) ${ }^{100}$ analysed 10 trials involving 3451 participants and found that probiotics reduced the number of participants experiencing acute URTI. Other studies, including two large cohort studies, found that probiotics reduced the duration and severity of influenza-like symptoms in children. ${ }^{102}{ }^{103}$ A community-based study in the USA showed that ingestion of probiotic-containing milk reduced the frequency of acute diarrhoeal illnesses by $24 \%$ and URTI by $18 \%$, but with no difference in rates of lower respiratory tract infections. ${ }^{101}$ A community-based study of children living in impoverished conditions in India tested milk fortified with $L$ casei Shirota compared with a nutrient drink in 3758 15 -year-old children. ${ }^{104}$ The frequency of acute diarrhoeal episodes was reduced by $14 \%$ in the probiotic group. Another study reported that a probiotic significantly shortened the duration of individual episodes of URTI (by almost 2 days) and reduced the severity of URTI symptoms even though the product had no effect on the frequency. ${ }^{105}$ Taken together, these studies suggest that probiotics in otherwise healthy individuals may reduce common infectious diseases.

For most of the conditions discussed, there is evidence suggesting benefit of probiotics. However, before definitive treatment or dietary management recommendations can be made, a systematic approach to evaluating the strength of evidence is needed to identify limitations in existing clinical studies. A clear definition of effective probiotic strain(s) and doses, identification of responding populations, quantification of the magnitude of expected effects, and characterisation of underlying microbiota deficiencies (microbes and/or their metabolites) are important for full implementation of probiotic interventions.

\section{CHALLENGES TO STUDYING HEALTH EFFECTS OF PROBIOTICS}

The findings from research reach their full potential when translated into effective products. Part of this process requires understanding the regulatory issues leading to technology transfer and commercialisation. This section addresses how to design human trials to be both scientifically meaningful and appropriate for corresponding product category regulations. Those designing clinical trials need to be mindful of recent regulatory actions pertinent to the probiotic field and of the potential challenges imposed by regulatory frameworks, especially in the USA and Europe. 


\section{Designing a clinical study on probiotics}

The value of well-controlled and well-designed human trials to elucidate probiotic efficacy is self-evident. However, the current heightened scrutiny from regulatory authorities on health benefit claims made on packaging and in advertising, combined with strict interpretations regarding what constitutes drug research, requires carefully weighing regulatory issues when designing and launching probiotic studies for food or supplement uses. Many issues worth addressing in the planning stages by both researchers and study sponsors are described in box 1 .

One fundamental issue in the concept of probiotics that is often not addressed in clinical trials is the importance of probiotic viability to the physiological benefit; non-viable controls are rarely used in studies. Although many of the mechanisms proposed for probiotic activity probably require growth and metabolism at the physiological site of action, confirmation of the requirement for viability through design of clinical studies with a non-viable control would clarify this issue. However, a non-viable product is not considered to be a probiotic, which by definition must be a live microbe. Such a product would fall under the more general term, 'pharmabiotic', which encompasses non-viable microbes and health-promoting substances derived from micro-organisms. ${ }^{48}$

\section{Effect of regulatory frameworks on probiotic development pathways}

The approach to marketing probiotic products is inextricably tied to the regulatory framework. These frameworks, although different in different countries worldwide, affect research approaches, communication strategies, product manufacturing and product labels. The importance of these regulatory matters in current times is reflected in some recent papers. ${ }^{106-109}$

A research path must stand up to scientific scrutiny, but also must comply with regulatory definitions of what constitutes appropriate research end points for specific product categories. The path for research on drugs is fairly clear. However, the path to provide evidence that will substantiate a health benefit claim for a food or dietary supplement is not as obvious. Many probiotic products are marketed as foods or dietary supplements, yet much of the research documenting health benefits is considered by regulatory authorities of some countries to be drug-use end points. Such research may not be considered appropriate to substantiate health benefits of food, as foods are not regulated as disease prevention or treatment measures, but only to support or maintain normal body functions or reduce the risk of disease in the general population. (The claim that a 'food' can treat or prevent disease turns it into a drug.) One challenge therefore is how to conduct meaningful studies to show that health is improved-or, even more challenging, maintained-in a healthy person? What does 'maintained' mean with respect to a study's primary outcome?

Communicating health benefits on probiotic products has emerged as a challenge for probiotic companies. For example, in Europe, no health benefit claims for a probiotic have been approved, apparently because the level of evidence does not meet the expectations of the regulatory authorities. Further, claims of disease risk reduction require demonstrated changes in commonly accepted risk factors for specific diseases. Changes in a clinical end point are not sufficient, and many of the diseases/disorders for which probiotics are being explored lack validated biomarkers. In the USA, claims worded as 'structure/function' claims (eg, 'this probiotic improves digestive
Box 1 Questions to consider before designing, conducting and reporting human studies on probiotics

- Will the study be performed among healthy subjects or in a disease population? Foods and dietary supplements are products generally intended for the healthy population. Therefore, trials designed to support claims about this category of products should be conducted on subjects who are representative of the healthy general population. If the target is a disease state, then the study must be performed in an appropriately selected study population representative of that disorder, and the study should conform to the standards of a pharmaceutical product.

- What is the regulatory approach to health benefit claims in your jurisdiction on the type of product you intend to market? Will the product be a food, supplement, drug or other? Efficacy standards, appropriate study end points, target populations and risk assessment all vary for the different categories of product, and the clinical research programme needs to reflect these differences.

- If seeking support for a claim for a probiotic food or supplement, what precisely is the claim and will it be supported by the study that you propose? The primary end point in the research study should be as close as possible to the benefit that will be communicated. Outcomes must be clear and measurable, and the study protocol should preferably involve a randomised, double-blind, placebo-controlled design. The selected placebo must be very similar to the probiotic food in terms of nutritional profile, taste, texture, colour and flavour, but should be devoid of the probiotic strains.

- Has the strain(s) of choice been adequately characterised, in terms of its genome, in vitro properties and in vivo activities, and ability to survive transit through the gastrointestinal tract? Thorough strain characterisation is critical to a full understanding of the substance being studied, and also to ensure the ease of repetition of the study by other research groups.

- Has the proposed formulation been shown to retain viability and efficacy for the duration of its proposed shelf life and in the environment in which it is likely to be marketed?

- Has a plausible rationale been developed for the use of this/ these particular strain/strains in this indication? Although a confirmed mechanism of action is not considered essential for functional food ingredients (or drugs, for that matter), a plausible rationale is preferred.

- Has the optimal target population been clearly defined for this particular probiotic and the specific outcome you plan to modify? If the target population is some subset of the general population for a food or supplement, it is important to document this.

- What dose will be tested? Is there any indication of an effective dose from previous studies? The dose used in the study must be high enough to confer the benefit, but not so high as to make the product commercially untenable. Since the product dose must match the dose in the human trial showing benefit, dose used in the study is an important consideration.

health') do not require approval, but must be substantiated nonetheless. Here the challenge is what types of studies support such a general claim. 
Despite the lack of clarity in how to substantiate claims, regulatory authorities in the USA have increased their scrutiny of structure/function claims, demanding that the claims meet regulatory standards for substantiation. A growing body of scientific evidence demonstrates that some probiotic foods or supplements may prevent or mitigate some diseases or illnesses. These products may be foods or supplements in the mind of consumers, but are considered drugs by regulators.

\section{THE FUTURE}

An association of microbiome alterations with a progression from health to disease seems clear. However, causality and reversal of disease in response to probiotic-induced microbiome changes still remain to be demonstrated. ${ }^{110} 111$ Until a healthy microbiome is clearly defined, providing a microbiological target for probiotic interventions, probiotic benefits must be described in the context of physiological or clinical improvement. Some promising immune and gastrointestinal clinical targets have been identified in this review, including paediatric rotavirus diarrhoea, antibiotic-associated diarrhoea, $C$ difficileassociated diarrhoea, ulcerative colitis, pouchitis, IBS, NEC and radiation enteritis. Beyond these, probiotic interventions with implications outside the gastrointestinal tract are increasingly recognised. Perhaps the most intriguing targets are focused on microbiota-influenced conditions of diabetes, the metabolic syndrome and obesity, where studies in animal models indicate functional involvement of the microbiota. To what extent targeted probiotic interventions affect these diseases is an area of active and evolving research.

In the future, probiotics developed to address microbiota-associated conditions will probably move beyond the micro-organisms commonly used as probiotics today. Genetically modified micro-organisms can provide epitopes for efficient oral vaccine delivery, improve vaccine or natural immune responses, or restore antigen-specific tolerance. ${ }^{112} 113$ Probiotic strains with altered cell surface components, such as lipoteichoic acid, provide a potential strategy for the treatment of inflammatory intestinal disorders. ${ }^{57}{ }^{114}$ The use of faecal transplants to replace dysbiotic bacterial communities with protective ones in order to manage $C$ difficile infections, IBD or IBS has met with some success. ${ }^{115}$ Recently, faecal microbiota transplant from non-diabetic donors infused into the duodenum of patients with the metabolic syndrome improved their insulin sensitivity, highlighting the broad potential of this intervention. ${ }^{116}$ However, cocktails of defined microbes imparting key functionalities may provide a more acceptable approach. Finally, specific strains with uniquely useful properties, such as Oxalobacter formigines (kidney stones), F prausnitzii (chronic gut inflammation), Bacteroidetes and Fusobacterium (cancer risk), should be more thoroughly studied in welldesigned clinical trials.

Probiotic interventions for extending the remission of IBD show promise for pouchitis and ulcerative colitis, but success with Crohn's disease will require new approaches. Identification of specific protective molecules, such as interleukin 10, ganglioside and trefoil factors, that can be engineered into probiotics for in situ release holds promise. ${ }^{112} 117$ More effective probiotic interventions for microbiota-associated conditions require a more complete understanding of the interactions between genetic, microbial and environmental influences within individuals. Such an approach will also facilitate the identification of subsets of patients most likely to respond to manipulations of the gut microbiota and the optimal agents to use in an individual subject.
Reducing the risk of disease with probiotics is promising, but validated biomarkers for many target diseases (such as allergy, infectious diarrhoea and IBS) are lacking. Consensus from the relevant research communities to define validated biomarkers would greatly advance this field. Measurement of meaningful physiological changes in healthy populations requires better definition as well. Identifying subjects on the edges of a normal physiological range, and intervening so as to move them closer to the median, may be a productive approach to research on healthy subjects. The effect of widespread use of safe and effective probiotic products on society-wide economic and quality-of-life indicators should be assessed with end points such as reduction of common infectious diseases in developing and developed nations. Such information could provide support for sustained research in this field.

\section{Key messages}

Clinical uses of probiotics

- While altered microbiota have been associated with obesity, the metabolic syndrome, non-alcoholic steatohepatitis, inflammatory bowel disease, irritable bowel syndrome (IBS), atherosclerosis, type 1 diabetes, autism, allergy, asthma and coeliac disease, a cause-and-effect relationship in the pathogenesis of these disorders in relation to the role of mutualistic micro-organisms remains to be defined.

- A key unanswered question is whether disease-prone microbiota can be remodelled to be more robust, resilient and disease-free with the use of probiotics as either a prevention or intervention strategy.

- Some probiotics can improve clinical outcomes for acute infectious diarrhoea, antibiotic-associated diarrhoea, necrotising enterocolitis, IBS, cancer therapies, pouchitis and possibly ulcerative colitis.

- Some probiotics deliver benefits for healthy individuals, such as reducing the risk of common infectious diseases and improving intestinal function.

- Probiotics probably function by altering the composition and/ or activities of the colonising microbiota and by direct interaction with the host through immune signalling mechanisms.

Acknowledgements We thank Elizabeth Kuvinka for designing and drawing the figure for this paper.

Contributors The authors collaborated on this paper after participating in a meeting covering these topics, but received no financial compensation for authorship. All contributed equally to the writing of the manuscript, and read and approved the final version.

Competing interests MES consults with numerous probiotic companies, but has no financial stake in any company. She is a member of the Danone Yakult US probiotics scientific board. FG is member of the Scientific Committee of Instituto Danone (Barcelona, Spain). RG is a member of the Danone Yakult US probiotics scientific board. PRH is a member of the Danone Yakult US probiotics scientific board. EMMO has served as a consultant, advisory board member and/or speaker for Movetis, Shire, Ironwood, Almirall, Janssen, Yakult, Procter and Gamble, Rhythm and Alimentary Health and has received research funding from Procter and Gamble, Merck, Alimentary Health and Norgine. He is a non-executive board member of, and shareholder in, Alimentary Health. His research is supported in part through a grant to the Alimentary Pharmabiotic Centre from Science Foundation, Ireland. RBS has served as a consultant, advisory board member and/or speaker for Dannon, Yakult, Procter \& Gamble and Salix. PMS serves on medical advisory boards for Abbott Nutrition, Mead Johnson Nutrition and Procter \& Gamble, has received research grants from Institut Rosell-Lallemand, is a stockholder in Antibe Therapeutics, and has received an honorarium from ILSI North America. EM is a member of the Danone 
International Advisory Board for Activia and the Danone Yakult US probiotics scientific board.

\section{Provenance and peer review Commissioned; externally peer reviewed.}

Open Access This is an Open Access article distributed in accordance with the Creative Commons Attribution Non Commercial (CC BY-NC 3.0) license, which permits others to distribute, remix, adapt, build upon this work non-commercially, and license their derivative works on different terms, provided the original work is properly cited and the use is non-commercial. See: http://creativecommons.org/ licenses/by-nc/3.0/

\section{REFERENCES}

1. Neish AS. Microbes in gastrointestinal health and disease. Gastroenterology [Research Support, N.I.H., Extramural Review] 2009;136:65-80.

2. Bron PA, van Baarlen P, Kleerebezem M. Emerging molecular insights into the interaction between probiotics and the host intestinal mucosa. Nat Rev Microbiol 2012;10:66-78

3. McNulty NP, Yatsunenko T, Hsiao A, et al. The impact of a consortium of fermented milk strains on the gut microbiome of gnotobiotic mice and monozygotic twins. Sci Trans/ Med [Research Support, N.I.H., Extramural] 2011;3:106ra.

4. Ringel Y, Quigley E, Lin H. Probiotics and gastrointestinal disorders. Am J Gastroenterol Supp/ 2012;1:34-40.

5. Spiller R, Aziz 0, Creed F, et al. Guidelines on the irritable bowel syndrome: mechanisms and practical management. Gut 2007;56:1770-98.

6. Quigley EM, Locke GR, Mueller-Lissner S, et al. Prevalence and management of abdominal cramping and pain: a multinational survey. Aliment Pharmacol Ther 2006;24:411-19.

7. Longstreth GF, Thompson WG, Chey WD, et al. Functional bowel disorders. Gastroenterology 2006;30:1480-91.

8. Moayyedi P, Ford AC. Symptom-based diagnostic criteria for irritable bowe syndrome: the more things change, the more they stay the same. Gastroenterol Clin North Am 2011;40:87-103.

9. European Food Safety Authority. Guidance on the scientific requirements for health claims related to gut and immune function. EFSA Journal 2011;9:1-12.

10. Kassinen A, Krogius-Kurikka L, Makivuokko H, et al. The fecal microbiota of irritable bowel syndrome patients differs significantly from that of healthy subjects. Gastroenterology 2007;133:24-33.

11. Saulnier DM, Riehle K, Mistretta TA, et al. Gastrointestinal microbiome signatures of pediatric patients with irritable bowel syndrome. Gastroenterology 2011;141:1782-91

12. Rajilic-Stojanovic M, Biagi E, Heilig HG, et al. Global and deep molecular analysis of microbiota signatures in fecal samples from patients with irritable bowel syndrome. Gastroenterology [Research Support, Non-U.S. Gov't] 2011;141:1792-801.

13. Jeffery IB, O'Toole PW, Ohman L, et al. An irritable bowel syndrome subtype defined by species-specific alterations in faecal microbiota. Gut [Research Support Non-U.S. Gov't] 2012;61:997-1006.

14. Jeffery IB, Quigley EM, Ohman L, et al. The microbiota link to irritable bowe syndrome: an emerging story. Gut Microbes 2012;3:572-6.

15. Carroll IM, Ringel-Kulka T, Keku TO, et al. Molecular analysis of the luminal- and mucosal-associated intestinal microbiota in diarrhea-predominant irritable bowel syndrome. Am J Physiol Gastrointest Liver Physiol [Research Support, N.I.H. Extramural] 2011;301:G799-807.

16. Rhee SH, Pothoulakis C, Mayer EA. Principles and clinical implications of the brain-gut-enteric microbiota axis. Nat Rev Gastroenterol Hepatol [Research Support, N.I.H., Extramural Review] 2009:6:306-14.

17. Kunze WA, Mao YK, Wang B, et al. Lactobacillus reuteri enhances excitability of colonic AH neurons by inhibiting calcium-dependent potassium channel opening. J Cell Mol Med 2009;13:2261-70.

18. Tillisch K, Labus J, Ebrat B, et al. Modulation of the brain-gut axis after 4-week intervention with a probiotic fermented dairy product. Gastroenterology 2012;142/5 Suppl 1):S-115

19. Moayyedi $\mathbf{P}$, Ford AC, Talley NJ, et al. The efficacy of probiotics in the treatment of irritable bowel syndrome: a systematic review. Gut 2010;59:325-32.

20. Clarke G, Cryan JF, Dinan TG, et al. Review article: probiotics for the treatment of irritable bowel syndrome-focus on lactic acid bacteria. Aliment Pharmacol Ther [Research Support, Non-U.S. Gov't Review] 2012;35:403-13.

21. Brenner DM, Moeller MJ, Chey WD, et al. The utility of probiotics in the treatment of irritable bowel syndrome: a systematic review. Am J Gastroenterol [Review] 2009;104:1033-49; quiz 50.

22. O'Mahony L, McCarthy J, Kelly P, et al. Lactobacillus and bifidobacterium in irritable bowel syndrome: symptom responses and relationship to cytokine profiles. Gastroenterology [Clinical Trial Randomized Controlled Trial Research Support, Non-U.S. Gov't] 2005:128:541-51.
23. Whorwell PJ, Altringer L, Morel J, et al. Efficacy of an encapsulated probiotic Bifidobacterium infantis 35624 in women with irritable bowel syndrome. Am J Gastroenterol [Multicenter Study Randomized Controlled Trial Research Support Non-U.S. Gov't] 2006;101:1581-90.

24. Black RE, Cousens S, Johnson HL, et al. Global, regional, and national causes of child mortality in 2008: a systematic analysis. Lancet [Comparative Study Research Support, Non-U.S. Gov't] 2010;375:1969-87.

25. Preidis GA, Hill C, Guerrant RL, et al. Probiotics, enteric and diarrheal diseases, and global health. Gastroenterology 2011;140:8-14.

26. Guerrant RL, Kosek M, Lima AA, et al. Updating the DALYs for diarrhoea disease. Trends Parasitol [News Research Support, Non-U.S. Gov't Research Support, U.S. Gov't, P.H.S.] 2002;18:191-3.

27. Guerrant RL, Oria RB, Moore SR, et al. Malnutrition as an enteric infectious disease with long-term effects on child development. Nutr Rev [Research Support, N.I.H., Extramural Research Support, Non-U.S. Gov't Review]. 2008;66:487-505.

28. Allen SJ, Martinez EG, Gregorio GV, et al. Probiotics for treating acute infectious diarrhoea. Cochrane Database Syst Rev 2010(11):CD003048.

29. Bernaola Aponte G, Bada Mancilla CA, Carreazo Pariasca NY, et al. Probiotics for treating persistent diarrhoea in children. Cochrane Database Syst Rev 2010(11): CD007401.

30. Preidis GA, Versalovic J. Targeting the human microbiome with antibiotics, probiotics, and prebiotics: gastroenterology enters the metagenomics era. Gastroenterology 2009;136:2015-31.

31. Guandalini S. Probiotics for prevention and treatment of diarrhea. J Clin Gastroenterol [Review]. 2011:45(Suppl):S149-53.

32. Floch $\mathbf{M H}$, Walker WA, Madsen K, et al. Recommendations for probiotic use-2011 update. J Clin Gastroenterol [Consensus Development Conference Practice Guideline Research Support, Non-U.S. Gov't] 2011;45(Suppl):S168-71.

33. Szajewska H, Ruszczynski M, Radzikowski A. Probiotics in the prevention of antibiotic-associated diarrhea in children: a meta-analysis of randomized controlled trials. J Pediatr 2006;149:367-72.

34. Parkes GC, Sanderson JD, Whelan K. The mechanisms and efficacy of probiotics in the prevention of Clostridium difficile-associated diarrhoea. Lancet Infect Dis 2009:9:237-44.

35. Hickson M, D'Souza AL, Muthu N, et al. Use of probiotic Lactobacillus preparation to prevent diarrhoea associated with antibiotics: randomised double blind placebo controlled trial. BMJ 2007;335:80.

36. Gao XW, Mubasher M, Fang $\mathrm{CY}$, et al. Dose-response efficacy of a proprietary probiotic formula of Lactobacillus acidophilus CL1285 and Lactobacillus case LBC80R for antibiotic-associated diarrhea and Clostridium difficile-associated diarrhea prophylaxis in adult patients. Am J Gastroenterol 2010;105:1636-41.

37. Scott RD II. The direct medical costs of healthcare-associated infections in U.S. hospitals and the benefits of prevention. Centers for Disease Control and Prevention. 2009; http://www.cdc.gov/HAl/pdfs/hai/Scott_CostPaper.pdf laccessed 25 Feb 2013).

38. Morrow LE, Kollef MH, Casale TB. Probiotic prophylaxis of ventilator-associated pneumonia: a blinded, randomized, controlled trial. Am J Respir Crit Care Med [Randomized Controlled Trial Research Support, N.I.H., Extramural Research Support, Non-U.S. Gov't] 2010;182:1058-64.

39. Hojsak I, Abdovic S, Szajewska H, et al. Lactobacillus GG in the prevention of nosocomial gastrointestinal and respiratory tract infections. Pediatrics 2010;125: e1171-7.

40. Mihatsch WA, Vossbeck S, Eikmanns B, et al. Effect of Bifidobacterium lactis on the incidence of nosocomial infections in very-low-birth-weight infants: a randomized controlled trial. Neonatology [Randomized Controlled Trial Research Support, Non-U.S. Gov't] 2010;98:156-63.

41. Honeycutt TC, El Khashab M, Wardrop RM III, et al. Probiotic administration and the incidence of nosocomial infection in pediatric intensive care: a randomized placebo-controlled trial. Pediatr Crit Care Med [Randomized Controlled Trial Research Support, Non-U.S. Gov't] 2007;8:452-8; quiz 64.

42. Szajewska H, Kotowska M, Mrukowicz JZ, et al. Efficacy of Lactobacillus GG in prevention of nosocomial diarrhea in infants. J Pediatr [Clinical Trial Multicenter Study Randomized Controlled Trial Research Support, Non-U.S. Gov't] 2001;138:361-5.

43. Mastretta E, Longo P, Laccisaglia A, et al. Effect of Lactobacillus GG and breast-feeding in the prevention of rotavirus nosocomial infection. $J$ Pediatr Gastroenterol Nutr [Clinical Trial Randomized Controlled Trial] 2002;35:527-31.

44. Szajewska H, Wanke M, Patro B. Meta-analysis: the effects of Lactobacillus rhamnosus GG supplementation for the prevention of healthcare-associated diarrhoea in children. Aliment Pharmacol Ther [Research Support, Non-U.S. Gov't] 2011;34:1079-87

45. Saavedra JM, Bauman NA, Oung I, et al. Feeding of Bifidobacterium bifidum and Streptococcus thermophilus to infants in hospital for prevention of diarrhoea and shedding of rotavirus. Lancet [Clinical Trial Randomized Controlled Trial Research Support, Non-U.S. Gov't Research Support, U.S. Gov't, P.H.S.] 1994;344:1046-9

46. Barraud D, Blard C, Hein F, et al. Probiotics in the critically ill patient: a double blind, randomized, placebo-controlled trial. Intensive Care Med [Randomized Controlled Trial Research Support, Non-U.S. Gov't] 2010;36:1540-7. 
47. Besselink MG, van Santvoort HC, Buskens E, et al. Probiotic prophylaxis in predicted severe acute pancreatitis: a randomised, double-blind, placebo-controlled trial. Lancet [Multicenter Study Randomized Controlled Trial Research Support, Non-U.S. Gov't] 2008;371:651-9.

48. Shanahan $\mathbf{F}$, Collins SM. Pharmabiotic manipulation of the microbiota in gastrointestinal disorders, from rationale to reality. Gastroenterol Clin North Am 2010;39:721-6

49. Sartor RB. Microbial influences in inflammatory bowel diseases. Gastroenterology 2008; 134:577-94.

50. Kruis W, Fric P, Pokrotnieks J, et al. Maintaining remission of ulcerative colitis with the probiotic Escherichia coli Nissle 1917 is as effective as with standard mesalazine. Gut [Clinical Trial Multicenter Study Randomized Controlled Trial] 2004; 53:1617-23.

51. Miele E, Pascarella F, Giannetti E, et al. Effect of a probiotic preparation (VSL\#3) on induction and maintenance of remission in children with ulcerative colitis. $\mathrm{Am} \mathrm{J}$ Gastroenterol [Randomized Controlled Trial] 2009;104:437-43.

52. Sood A, Midha V, Makharia GK, et al. The probiotic preparation, VSL\#3 induces remission in patients with mild-to-moderately active ulcerative colitis. Clin Gastroenterol Hepatol [Multicenter Study Randomized Controlled Trial Research Support, Non-U.S. Gov't] 2009;7:1202-9, 9 e1.

53. Tursi A, Brandimarte G, Papa A, et al. Treatment of relapsing mild-to-moderate ulcerative colitis with the probiotic VSL\#3 as adjunctive to a standard pharmaceutical treatment: a double-blind, randomized, placebo-controlled study. Am J Gastroenterol [Multicenter Study Randomized Controlled Trial Research Support, Non-U.S. Gov't] 2010;105:2218-27.

54. McGovern DP, Jones MR, Taylor KD, et al. Fucosyltransferase 2 (FUT2) non-secretor status is associated with Crohn's disease. Hum Mol Genet 2010;19:3468-76.

55. Yan F, Polk D. Lactobacillus rhamnosus GG-an updated strategy to use microbial products to promote health. Functional Food Rev 2012;4:41-8.

56. Round JL, Lee SM, Li J, et al. The Toll-like receptor 2 pathway establishes colonization by a commensal of the human microbiota. Science 2011;332: 974-7

57. Mohamadzadeh M, Pfeiler EA, Brown JB, et al. Regulation of induced colonic inflammation by Lactobacillus acidophilus deficient in lipoteichoic acid. Proc Nat/ Acad Sci USA 2011;108(Suppl 1):4623-30.

58. Whitehead KJ, Schmitz JM, Carroll I, et al. Ingestion of dietary carbohydrates influences the aggressiveness of colitis and intestinal microbial composition in IL-10-/- mice. Gastroenterology 2011;138:A51.

59. Matsuura M, Liu B, Carroll I, et al. Selective alteration of growth and virulence for iron-dependent bacterial species; Possible novel mechanisms of detrimental effects of dietary iron on intestinal inflammation. Gastroenterology 2011;138:A734

60. Neu J, Walker WA. Necrotizing enterocolitis. N Engl J Med [Review] 2011;364:255-64.

61. Morowitz MJ, Poroyko V, Caplan M, et al. Redefining the role of intestinal microbes in the pathogenesis of necrotizing enterocolitis. Pediatrics [Review] 2010;125:777-85.

62. Wang Y, Hoenig JD, Malin KJ, et al. 16S rRNA gene-based analysis of fecal microbiota from preterm infants with and without necrotizing enterocolitis. ISME J [Research Support, N.I.H., Extramural] 2009;3:944-54.

63. Mai V, Young CM, Ukhanova $\mathrm{M}$, et al. Fecal microbiota in premature infants prior to necrotizing enterocolitis. PLoS One 2011:6:e20647.

64. Nanthakumar NN, Fusunyan RD, Sanderson I, et al. Inflammation in the developing human intestine: A possible pathophysiologic contribution to necrotizing enterocolitis. Proc Natl Acad Sci USA 2000;97:6043-8.

65. Deshpande G, Rao S, Patole $S$, et al. Updated meta-analysis of probiotics for preventing necrotizing enterocolitis in preterm neonates. Pediatrics [Meta-Analysis] 2010;125:921-30.

66. Awad H, Mokhtar H, Imam SS, et al. Comparison between killed and living probiotic usage versus placebo for the prevention of necrotizing enterocolitis and sepsis in neonates. Pak J Biol Sci 2010;13:253-62.

67. Thomas DW, Greer FR. Probiotics and prebiotics in pediatrics. Pediatrics [Review] 2010;126:1217-31.

68. Szajewska H, Guandalini S, Morelli L, et al. Effect of Bifidobacterium animalis subsp lactis supplementation in preterm infants: a systematic review of randomized controlled trials. J Pediatr Gastroenterol Nutr [Meta-Analysis Research Support, Non-U.S. Gov't Review] 2010:51:203-9.

69. Alfaleh K, Anabrees J, Bassler D, et al. Probiotics for prevention of necrotizing enterocolitis in preterm infants. Cochrane Database Syst Rev [Meta-Analysis Review] 2011(3):CD005496.

70. Vannucci L, Stepankova R, Kozakova H, et al. Colorectal carcinogenesis in germ-free and conventionally reared rats: different intestinal environments affect the systemic immunity. Int J Oncol 2008;32:609-17.

71. Sears CL, Pardoll DM. Perspective: alpha-bugs, their microbial partners, and the link to colon cancer. J Infect Dis [Research Support, N.I.H., Extramural Research Support, Non-U.S. Gov't] 2011:203:306-11.

72. Chen W, Liu F, Ling Z, et al. Human intestinal lumen and mucosa-associated microbiota in patients with colorectal cancer. PLoS One 2012:7:e39743.
73. Sobhani I, Tap J, Roudot-Thoraval F, et al. Microbial dysbiosis in colorectal cancer (CRC) patients. PLoS One 2011;6:e16393.

74. Marchesi JR, Dutilh BE, Hall N et al. Towards the human colorectal cance microbiome. PLoS One [Research Support, Non-U.S. Gov't] 2011;6:e20447.

75. Castellarin $\mathbf{M}$, Warren RL, Freeman JD, et al. Fusobacterium nucleatum infection is prevalent in human colorectal carcinoma. Genome Res [Research Support, Non-U.S. Gov't] 2012;22:299-306.

76. Saikali J, Picard C, Freitas M, et al. Fermented milks, probiotic cultures, and colon cancer. Nutr Cancer 2004;49:14-24.

77. Zhu Y, Michelle Luo T, Jobin C, et al. Gut microbiota and probiotics in colon tumorigenesis. Cancer Lett 2011;309:119-27.

78. Azcarate-Peril MA, Sikes M, Bruno-Barcena JM. The intestinal microbiota, gastrointestinal environment and colorectal cancer: a putative role for probiotics in prevention of colorectal cancer? Am J Physiol Gastrointest Liver Physiol [Review] 2011;301:G401-24.

79. Rowland IR. The role of the gastrointestinal microbiota in colorectal cancer. Curr Pharm Des 2009;15:1524-7.

80. Pearson JR, Gill Cl, Rowland IR. Diet, fecal water, and colon cancer-development of a biomarker. Nutr Rev 2009;67:509-26.

81. Worthley DL, Le Leu RK, Whitehall VL, et al. A human, double-blind, placebo-controlled, crossover trial of prebiotic, probiotic, and synbiotic supplementation: effects on luminal, inflammatory, epigenetic, and epithelial biomarkers of colorectal cancer. Am J Clin Nutr 2009:90:578-86.

82. Rafter J, Bennett M, Caderni G, et al. Dietary synbiotics reduce cancer risk factors in polypectomized and colon cancer patients. Am J Clin Nutr [Randomized Controlled Trial Research Support, Non-U.S. Gov't] 2007;85:488-96.

83. Ishikawa H, Akedo I, Otani T, et al. Randomized trial of dietary fiber and Lactobacillus casei administration for prevention of colorectal tumors. Int J Cancer 2005;116:762-7.

84. Pala V, Sieri S, Berrino F, et al. Yogurt consumption and risk of colorectal cancer in the Italian European prospective investigation into cancer and nutrition cohort. Int $J$ Cancer 2011:129:2712-9.

85. Crawford PA, Gordon Jl. Microbial regulation of intestinal radiosensitivity. Proc Natl Acad Sci USA 2005;102:13254-9.

86. Xue H, Field CJ, Sawyer MB, et al. Prophylactic ciprofloxacin treatment prevented high mortality, and modified systemic and intestinal immune function in tumour-bearing rats receiving dose-intensive CPT-11 chemotherapy. Br J Cancer 2009;100:1581-8.

87. Ciorba MA, Stenson WF. Probiotic therapy in radiation-induced intestinal injury and repair. Ann N Y Acad Sci 2009;1165:190-4

88. Osterlund P, Ruotsalainen T, Korpela R, et al. Lactobacillus supplementation for diarrhoea related to chemotherapy of colorectal cancer: a randomised study. $\mathrm{Br} \mathrm{J}$ Cancer 2007;97:1028-34.

89. Chitapanarux I, Chitapanarux T, Traisathit P, et al. Randomized controlled trial of live lactobacillus acidophilus plus bifidobacterium bifidum in prophylaxis of diarrhea during radiotherapy in cervical cancer patients. Radiat Oncol 2010;5:31.

90. Delia $\mathbf{P}$, Sansotta G, Donato V, et al. Use of probiotics for prevention of radiation-induced diarrhea. Tumori 2007; 93:(Suppl) 1-6.

91. Movsas B, Vikram B, Hauer-Jensen M, et al. Decreasing the adverse effects of cancer therapy: National Cancer Institute guidance for the clinical development of radiation injury mitigators. Clin Cancer Res 2011;17:222-8.

92. Bjorksten B, Sepp E, Julge K, et al. Allergy development and the intestinal microflora during the first year of life. J Allergy Clin Immunol [Research Support, Non-U.S. Gov't] 2001;108:516-20.

93. Dominguez-Bello MG, Costello EK, Contreras M, et al. Delivery mode shapes the acquisition and structure of the initial microbiota across multiple body habitats in newborns. Proc Natl Acad Sci USA [Research Support, N.I.H., Extramural Research Support, Non-U.S. Gov't] 2010;107:11971-5.

94. Shane AL, Cabana MD, Vidry S, et al. Guide to designing, conducting, publishing and communicating results of clinical studies involving probiotic applications in human participants. Gut Microbes 2010;1:243-53.

95. Folster-Holst R. Probiotics in the treatment and prevention of atopic dermatitis. Ann Nutr Metab 2010;57:16-19.

96. Skoner DP. Allergic rhinitis: definition, epidemiology, pathophysiology, detection, and diagnosis. J Allergy Clin Immunol 2001;108(1 Suppl):S2-8.

97. Mannheimer SB, Mukherjee R, Hirschhorn LR, et al. The CASE adherence index: a novel method for measuring adherence to antiretroviral therapy. AIDS Care 2006;18:853-61.

98. Stecher B, Hardt WD. Mechanisms controlling pathogen colonization of the gut. Curr Opin Microbiol [Research Support, Non-U.S. Gov't Review] 2011:14:82-91.

99. Borchers AT, Selmi C, Meyers FJ, et al. Probiotics and immunity. J Gastroenterol [Review] 2009:44:26-46.

100. Hao 0, Lu Z, Dong BR, et al. Probiotics for preventing acute upper respiratory tract infections. Cochrane Database Syst Rev [Meta-Analysis Review] 2011(9): CD006895.

101. Merenstein D, Murphy M, Fokar A, et al. Use of a fermented dairy probiotic drink containing Lactobacillus casei (DN-114 001) to decrease the rate of illness in kids: the DRINK study. A patient-oriented, double-blind, cluster-randomized, 
placebo-controlled, clinical trial. Eur J Clin Nutr [Randomized Controlled Trial Research Support, Non-U.S. Gov't] 2010;64:669-77.

102. Sazawal S, Dhingra U, Hiremath G, et al. Prebiotic and probiotic fortified milk in prevention of morbidities among children: community-based, randomized, double-blind, controlled trial. PLoS One [Randomized Controlled Trial Research Support, Non-U.S. Gov't] 2010;5:e12164.

103. Leyer GJ, Li S, Mubasher ME, et al. Probiotic effects on cold and influenza-like symptom incidence and duration in children. Pediatrics [Comparative Study Randomized Controlled Trial Research Support, Non-U.S. Gov't] 2009;124 e172-9.

104. Sur D, Manna B, Niyogi SK, et al. Role of probiotic in preventing acute diarrhoea in children: a community-based, randomized, double-blind placebo-controlled field trial in an urban slum. Epidemiol Infect [Randomized Controlled Trial Research Support, Non-U.S. Gov't] 2011;139:919-26.

105. de Vrese M, Winkler P, Rautenberg $P$, et al. Probiotic bacteria reduced duration and severity but not the incidence of common cold episodes in a double blind, randomized, controlled trial. Vaccine [Randomized Controlled Trial] 2006;24:6670-4.

106. Guarner F, Sanders ME, Gibson G, et al. Probiotic and prebiotic claims in Europe: seeking a clear roadmap. Br J Nutr 2011 Jun 21:1-3.

107. Klein M, Sanders ME, Duong T, et al. Probiotics: from bench to market. Ann N Y Acad Sci 2010;1212(Suppl 1):E1-14.

108. Sanders ME, Levy DD. The science and regulations of probiotic food and supplement product labeling. Ann N Y Acad Sci 2011;1219(Suppl 1):E1-E23.

109. Degnan FH. Clinical studies involving probiotics: when FDA's investigational new drug rubric applies-and when it may not. Gut Microbes 2012;3.
110. Gareau MG, Sherman PM, Walker WA. Probiotics and the gut microbiota in intestinal health and disease. Nat Rev Gastroenterol Hepatol 2010;7:503-14.

111. Bäckhed F, Fraser $C$, Ringel $Y$, et al. Defining a healthy human gut microbiome: current concepts, future directions, and clinical applications. Cell Host Microbe 2012;12:611-22.

112. Focareta A, Paton JC, Morona $\mathrm{R}$, et al. A recombinant probiotic for treatment and prevention of cholera. Gastroenterology 2006;130:1688-95.

113. Takiishi T, Korf H, Van Belle TL, et al. Reversal of autoimmune diabetes by restoration of antigen-specific tolerance using genetically modified Lactococcus lactis in mice. J Clin Invest [Research Support, Non-U.S. Gov't] 2012;122:1717-25.

114. Lebeer S, Vanderleyden J, De Keersmaecker SC. Host interactions of probiotic bacterial surface molecules: comparison with commensals and pathogens. Nat Rev Microbiol 2010;8:171-84

115. Aroniadis OC, Brandt LJ. Fecal microbiota transplantation: past, present and future. Curr Opin Gastroenterol 2013;29:79-84.

116. Vrieze A, Van Nood E, Holleman F, et al. Transfer of intestinal microbiota from lean donors increases insulin sensitivity in individuals with metabolic syndrome. Gastroenterology 2012;143:913-16 e7.

117. Wells JM, Mercenier A. Mucosal delivery of therapeutic and prophylactic molecules using lactic acid bacteria. Nat Rev Microbiol [Research Support, Non-U. S. Gov't Review] 2008:6:349-62.

118. Atarashi K, Tanoue T, Shima T, et al. Induction of colonic regulatory $T$ cells by indigenous Clostridium species. Science 2011;331:337-41.

119. Sartor RB. Genetics and environmental interactions shape the intestinal microbiome to promote inflammatory bowel disease versus mucosal homeostasis. Gastroenterology 2010;139:1816-19. 